\title{
Rhétorique administrative, stratégies discursives et mauvaise nouvelle
}

\author{
Céline Beaudet \\ Département des lettres et communications \\ Université de Sherbrooke
}

In argumentative discourse, writers strategize to present the reader with a vision of the world that appears to have the credibility of truth. This article analyses a corpus of annual reports and examines the discursive strategies that writers employ to construct the image of "truth" that supports and "justifies" a poor financial performance.

Le discours argumentatif prend forme dans le langage, instrument de symbolisation, qu'un énonciateur s'approprie pour rendre compte de sa schématisation du monde et pour convaincre un destinataire qu'il dit vrai. Nous examinons ici les stratégies discursives à l'oeuvre dans un corpus de rapports annuels, de manière à construire l'effet de sens vérité recherché par l'énonciateur, alors qu'il doit justifier de mauvais résultats financiers.

ARGUMENTATION ET POUVOIR SONT AU COEUR DES RELATIONS HUMAINES : en société, tout est enjeu, tout est à définir en fonction des intérêts de chacun et des groupes d'influence, qui se disputent les différents pouvoirs d'une communauté. Le discours argumentatif est le lieu de médiation de ces intérêts souvent contradictoires, tout au moins lorsqu'il n'est pas remplacé par des actes violents. Ce qui fait dire à Georges Vignaux (1978 : 137) que, «Le discours du pouvoir passe par le pouvoir du discours.» L'un configure l'autre, et vice versa : l'argumentation, construite en fonction d'une stratégie de pouvoir, trouve son expression privilégiée dans le discours, qui légitimera subséquemment le pouvoir qu'il a contribué à façonner.

L'ensemble de ces manipulations de nature symbolique laisse des traces apparentes dans le discours. Quelqu'un parle, se manifeste dans tout discours. Entre la réalité et le discours argumentatif, donc, se glisse irrémédiablement un énonciateur ayant pour but d'établir, d'édifier des faits et des vérités, de les 
valider, de les apprécier au profit de l'entendement d'un destinataire qu'il cherche à influencer. Le discours argumentatif, comme discours de pouvoir, a des visées pragmatiques. Il s'agit d'abord de faire croire pour ensuite faire faire, ce qui en fait un outil de pouvoir, comme le souligne Chaim Perelman $(1970: 25)$ :

"Toute argumentation vise, en effet, à un changement dans le chef des auditeurs, qu'il s'agisse de modifier les thèses mêmes auxquelles ils adhèrent ou simplement l'intensité de cette adhésion, mesurée par les conséquences ultérieures qu'elle tend à produire dans l'action. La perspective de l'argumentation ne permet pas comme celle de la démonstration de séparer entièrement la pensée de l'action et l'on comprend que l'exercice de l'argumentation soit tantôt favorisé, tantôt empêché, et souvent réglementé par ceux qui détiennent dans la société le pouvoir ou l'autorité."

Le discours argumentatif est, dans une perspective que l'on pourrait qualifier de darwiniste, la meilleure réponse que l'humanité ait trouvée (jusqu'ici) pour résoudre le conflit permanent entre le désir d'énoncer la vérité sur le monde, l'incapacité cognitive de saisir la complexité d'un monde en mouvance perpétuelle et de la traduire en discours, et la velléité de chaque locuteur de ramener ses préoccupations propres dans le discours et de faire en sorte qu'elles apparaissent inscrites dans la vérité du monde, tout en effaçant les traces de cette manipulation.

Comme le résume Pierre Oléron (1993:12) :

«L'argumentation est théoriquement subordonnée à la vérité, qu'elle est censée respecter et - bien plus - contribuer à établir. Mais cette préoccupation se trouve en conflit avec le souci de réussite et d'efficacité. [...] on peut s'en indigner, toujours au nom de vues idéalistes. Mais dans nombre de cas, sinon dans la totalité, contrairement à la théorie cartésienne de l'évidence, la vérité n'est pas l'objet d'une expérience spécifique, où elle apparaîtrait avec une étiquette qui la désigne sans équivoque. Tout ce qui peut être proposé est une certaine lecture de faits, à laquelle une autre lecture peut être opposée, elle aussi plausible et justifiee par des arguments.»

En ce sens, nous souscrivons entièrement à la définition de KerbratOrecchioni (1981:55), pour qui argumenter, «c'est modéliser l'univers par 
le truchement du langage.» Et nous ajoutons : dans le but d'exercer une influence pragmatique sur un destinataire, car l'argumentation est un outil de pouvoir.

Comme cette modélisation est orientée vers un destinataire, elle doit donc constamment inclure une justification de ses bases logico-discursives, car tel est le point de départ de tout le processus : il n'y a pas de pouvoir du discours si l'énonciateur n'est pas crédible. Le discours argumentatif prend forme dans le langage, instrument de symbolisation, qu'un énonciateur s'approprie pour rendre compte de sa schématisation du monde et convaincre un destinataire qu'il dit vrai. Il est donc postulé ici que la vérité est un effet de sens du discours, c'est-à-dire le résultat d'opérations discursives qu'il est possible de décrire, ce que nous chercherons à faire dans un corpus de trois messages d'introduction de rapports annuels, signés par la direction des entreprises suivantes : Hydro-Québec, Bell Canada et Seagram.

Ces messages ont un point en commun : ils sont centrés sur la justification d'une mauvaise nouvelle, soit la baisse des bénéfices de l'entreprise dans un contexte paradoxal de croissance des ventes. La haute direction de ces trois entreprises s'exerce donc à convaincre le destinataire du discours que ses décisions administratives sont les bonnes, malgré un échec apparent. Elle cherche aussi à signifier au destinataire que ses paroles sont vraies.

Il s'agit de discours de pouvoir, orientés vers leur effet pragmatique : les destinataires doivent renouveler leur confiance dans les directions de ces entreprises. Comme le résume bien Sophie Moirand (1995: 90), «Faire croire, faire savoir et faire agir ou réagir l'autre : telles sont les fonctions mises en oeuvre dans la communication externe des entreprises."

Nous nous intéresserons donc ici à trois stratégies ou opérations discursives qui s'entremêlent dans le discours de manière à construire l'effet de sens vérité recherché par l'énonciateur. «Tout sujet langagier, pour s'engager dans un acte de langage [...], doit résoudre le problème de savoir comment occuper l'espace de parole, écrit Patrick Charaudeau. Il devra donc d'une manière ou d'une autre légitimer ou justifier sa prise de parole.» (1995: 109)

En premier lieu, nous examinerons la construction de l'objet thématique, c'est-à-dire la dénomination de l'objet du discours, ses caractéristiques, les propriétés que l'énonciateur lui attribue et qui orienteront son exploitation dans l'économie globale du discours. "Celui qui énonce est celui qui pose le thème à traiter, qui établit frontière entre ce qu'il faut et ne faut pas considérer, qui asserte donc l'existence d'une certaine situation avec 
des objets qu'il choisit pour les y inclure, en les affectant de propriétés qui vont les montrer selon un certain éclairage déterminé." (Vignaux, 1988: 30)

La thématisation, c'est cette activité de dénomination, associée à la prédication, autre activité langagière qui consiste à doter un objet d'attributs, de qualités. Le résultat est l'objet thématisé dans le discours, dont la configuration est le signe d'un choix énonciatif; et dans un discours argumentatif, ce choix est fonction de la volonté de l'énonciateur de faire croire pour faire faire.

Nous nous intéresserons, en deuxième lieu, aux manifestations lexicales de la présence d'un énonciateur juge de son propre discours. En effet, l'énonciateur, en plus de thématiser un objet qu'il a construit dans un but persuasif, intervient librement dans son discours et en évalue la portée, la vérité, l'importance en regard de valeurs qui apparaissent comme vraies parce que, essentiellement, elles relèvent du discours social ambiant considéré comme acceptable : la force de l'argumentation tient à ses précédents, comme le fait valoir Perelman (1970 : 23).

En dernier lieu, dans notre analyse, nous considérerons la macro-structure logique du discours comme une stratégie de l'énonciateur. Dans les trois exemples retenus, le modèle narratif (le récit et son déroulement virtuel en quatre phases : transmission des valeurs, acquisition de la compétence, performance et sanction) domine dans un cas et le modèle argumentatif (variantes du plan polémique) caractérise les deux autres. La structuration du discours relève, dans notre perspective, d'une intention de stabiliser des notions autrement floues : l'une des manières d'y parvenir consiste à édifier une structure logique qui facilite la lecture de relations limpides, arrêtées, entre des énoncés s'appuyant sur des faisceaux de significations beaucoup plus fuyants que ce qui est souhaité par le discours. Or ce qui est clair est perçu comme vrai. La clarté, la vérité du discours tiennent à cet effort de l'énonciateur de stabiliser l'univers de sens que son discours construit, comme nous allons le constater dans les exemples qui suivent.

\section{Hydro-Québec, Rapport annuel, 1994}

Le rapport annuel d'Hydro-Québec, en 1994, comprend une mauvaise nouvelle : les ventes d'électricité sont en hausse mais le bénéfice net de l'entreprise est en baisse de 13\%. La lettre d'introduction du document, signée par le président du Conseil et par le président et chef de l'exploitation de la société d'État, s'étale sur trois pages et demie, dont une demie est consacrée à l'évaluation du bilan de l'année. Les trois autres pages permettent à la di- 
rection d'esquisser les grandes lignes de sa stratégie de développement pour les années qui viennent.

La lettre commence ainsi : «Tout au long de 1994, la performance d'Hydro-Québec s'est avérée plus que satisfaisante." L'objet thématisé dans le discours est identifié, d'entrée de jeu, comme la performance, ce qui est l'objet attendu dans un rapport annuel. Toutefois, cet objet correspond à un concept flou et le discours se charge de le préciser : la performance de l'entreprise est désignée ici comme équivalente de la notion de service à la clientèle. Cette notion prend forme grâce à une énumération : «la qualité du service, des actions rapides, la continuité du service et la sécurité.»

Le deuxième paragraphe poursuit le développement thématique de l'objet performance, défini comme service à la clientèle. Le sujet de la performance est désigné comme "nos employées et employés," dont l'exploit consiste à avoir vaincu la nature et ses obstacles, prenant la forme d'orages, de vents violents et de tempêtes de neige. Les obstacles sont tous fortement modalisés : les très grands froids, les vents violents, les très nombreux orages.

La narrativisation succincte de l'objet performance défini comme service à la clientèle permet de projeter l'acteur qu'est la direction de l'entreprise dans un rôle positif de co-évaluateur, accréditant les dires de la clientèle. "D'ailleurs, dans les pages qui suivent, nos clients témoignent de leur satisfaction," peut-on lire dès le premier paragraphe de la lettre. Il est clair qu'en se faisant l'écho de l'évaluation de la clientèle, la direction accrédite ses propres dires. Cette procédure est l'équivalent de l'argument d'autorité, dans un contexte de communication externe des entreprises.

Dans le troisième paragraphe, chapeauté d'un intertitre en caractère gras se lisant comme suit : Une gestion financière serrée, l'information négative quant à la baisse de bénéfices est révélée. L'objet thématisé n'est plus le même : la performance est maintenant déterminée par l'énoncé "gestion financière.» Voici l'énoncé de la première phrase : «Les produits de nos ventes ont connu une croissance appréciable en 1994, particulièrement dans le secteur industriel.»

Personne ne semble s'exprimer ici, ce qui n'était pas le cas lorsque la direction retourne vers elle le jugement favorable qu'elle prête à la clientèle. Cette stratégie est reprise tout au long du texte, les sujets prédiqués étant le plus souvent des concepts abstraits. Ainsi, la performance s'avère, le progrès touche, les facteurs jouent, les produits de ventes ont connu une croissance, l'accroissement de la charge d'amortissement a entraîné, etc. Tous ces exemples tendent à démontrer que personne n'agit dans cette entreprise. Les 
choses sont, comme la Terre tourne. En d'autres termes, les jugements d'évaluation sont présentés, le plus souvent, comme des jugements d'observation.

L'organisation logique du texte est ici une trace énonciative apparente. En substance, il s'agit de mettre l'accent sur la hausse des ventes, dont le rapport concède qu'elle est assortie d'une baisse du bénéfice net attribuable à des pressions immédiatement qualifiées de temporaires, ce qui permet de conclure le paragraphe par un jugement euphorique pris en charge, cette fois-ci, par le sujet animé qu'est la direction : "Nous demeurons donc résolument optimistes quant à une amélioration au cours des prochaines années.»

Lélément négatif de l'information, pourtant essentiel ici, est présenté comme secondaire dans cette analyse des résultats, car il n'est qu'une remarque concessive dans un raisonnement orienté vers la conclusion. Ce qui tend à s'interpréter comme ceci : nos ventes sont en hausse, donc nous sommes optimistes, malgré une baisse temporaire de nos bénéfices.

On constate que, pour présenter la mauvaise nouvelle, le discours s'appuie sur une structure argumentative plutôt que narrative : plutôt que de faire le récit des actions dont le résultat est somme toute peu avantageux pour l'image de l'entreprise, l'énonciateur choisit de porter un jugement qu'il argumente en ne retenant de la réalité que des faits lui permettant de corroborer ses dires.

La mise en verbe du jugement conclusif de l'argumentation traduit cette absence de préoccupation quant à l'importance de voir les bénéfices chuter : «nous demeurons,» lit-on, ce qui signale une absence de perturbation, un état stationnaire de satisfaction, désigné par l'adjectif appréciatif "optimistes,» surmodalisé par l'adverbe "résolument." Le discours est ici fortement modalisé.

Plusieurs stratégies sont mises à profit par l'énonciateur pour accréditer son rôle d'autorité dans l'entreprise, car tel est l'enjeu principal de cette prise de parole : légitimer sa position de pouvoir par un bilan favorable des actions entreprises pendant l'année (en dépit de données défavorables).

Ainsi, on remarque que l'entrée en matière est agressive : il s'agit de conclure sans d'abord démontrer. Autre stratégie : l'énonciateur du jugement se légitime en faisant porter, en partie, sa conclusion par un énonciateur extérieur, ici la clientèle. La performance faisant l'objet de l'évaluation est alors définie en fonction de ce nouvel énonciateur : puisque le juge auxiliaire est désigné comme étant la clientèle, alors la performance thématisée dans le 
discours devient le service à la clientèle (et non pas le bilan financier, que l'on pourrait, sauf erreur, désigner comme l'objet thématique attendu dans ce type d'écrit administratif).

On constate par ailleurs que, lorsque l'objet thématique devient la gestion de l'entreprise, l'énonciateur s'efface derrière un discours apparemment impersonnel.

L'objet performance est fortement modalisé sur l'échelle du lexique d'appréciation. On pourrait parler de frénésie énonciatrice, tant les adverbes de jugement, les quantifieurs, les superlatifs abondent. En ce sens, le jugement conclusif tient le lecteur prisonnier comme dans une toile d'araignée. Il est martelé, sans retenue, et ne cède jamais la place au doute, à la critique, au questionnement. Le jugement est limpide et simple comme la réalité construite pour le soutenir, ce qui traduit bien la réalité du discours argumentatif.

\section{Bell Canada, Rapport annuel, 1995}

Chez Bell Canada, les bénéfices sont en baisse de 30\% en 1995. L'objet thématisé dans le discours de direction, d'entrée de jeu, n'est pas la performance accomplie mais la performance à venir, comme le fait voir l'incipit : «Bell Canada et l'industrie des télécommunications s'apprêtent à livrer une grande bataille : la bataille de la concurrence mondiale."

Comme il s'agit d'un rapport annuel et que l'objet normalement prédiqué est la performance de l'année écoulée, ce choix thématique est à verser dans la stratégie rhétorique. L'incipit, par sa position privilégiée, est le lieu où l'objet thématique est posé, déterminé de manière à ce que le discours en exploite les avenues de sens suggérées par le choix associatif des mots utilisés. Bell Canada annonce ses couleurs : le rapport annuel de 1995 entretient le lecteur de l'année 1996.

La stratégie tient au fait que le jugement favorable sur l'entreprise doit être associé aux résultats, et que ces résultats n'appartiennent qu'à un avenir projeté. L'enjeu sera donc ici de diminuer le jugement d'incertitude qui accompagne l'exercice d'anticipation auquel le rapport annuel convie ses lecteurs.

Pour revenir à la désignation de l'objet thématisé dans le discours, Bell Canada le détermine par l'énoncé : la bataille de la concurrence mondiale. Encore une fois, la performance de l'entreprise n'est pas, apparemment, de vendre un produit (ici, les produits et services multiples d'une gigantesque entreprise de téléphonie), mais d'oeuvrer à obtenir un jugement favorable. 
Ici, ce jugement couronnera une victoire anticipée : «Les 36 prochains mois décideront de notre victoire dans le marché féroce des télécoms."

Étant donné cette orientation prospective du rapport, l'objet année écoulée ne fait surface qu'au quatrième paragraphe du message de la direction. Les éléments rapportés au passé composé et présentés dans une structure d'énumération donnent une impression d'ensemble fini, contrôlé et complet. Voici ce que l'entreprise a fait pour atteindre les objectifs énoncés ci-dessus et ces actions s'inscrivent dans une liste qui leur conferent un sens d'entreprise achevée, sur laquelle l'énonciateur a plein contrôle. Tout en conservant une enveloppe narrative globale pour son discours, l'énonciateur met l'accent sur la requalification du sujet. Cette stratégie discursive a une valeur argumentative certaine dans l'économie du discours de validation.

Les actions énumérées sont présentées comme finies, grâce au passé composé, ce qui atténue la valeur d'incertitude portée par les verbes utilisés pour présenter ces actions : «nous avons mis en oeuvre, dit le rapport, nous avons commencé, nous avons ralenti ..." En regardant de près cette liste d'actions, on constate qu'elle se constitue en instrument de validation d'un jugement en raison de la structure par énumération qui l'enveloppe et de la temporalité des verbes. La structure est renforcée par un dispositif de marqueurs logiques : nous avons, nous avons, nous avons aussi et enfin, nous sommes.

La structure englobante du discours est de type narratif : il s'agit de raconter comment l'entreprise est en train d'évoluer. En répartissant les segments du discours dans cette structure, il ressort que la dominante est la qualification du sujet, développée dans une structure par addition. Les verbes modaux abondent : Bell Canada veut faire, Bell Canada doit faire et Bell Canada veut encore faire, elle doit aussi être, tout cela pour ensuite accomplir cette performance qui se résume par vaincre la concurrence mondiale. Pourtant, le sujet est en régression, il doit se requalifier pour accomplir ce qu'il est supposé d'accomplir, soit vendre des produits téléphoniques avec profit.

Cette requalification du sujet se fait par l'énumération des actions futures qu'il entreprendra, donc une projection de performances dont le résultat anticipé est favorable. La requalification passe également par une redéfinition des valeurs du sujet, au nom desquelles il agira avec succès.

Il est donc clair que l'énonciateur du discours est ici en train de valider une performance non accomplie. L'accompli n'est pas évalué comme tel, les résultats obtenus ne sont pas des résultats, c'est-à-dire l'aboutissement d'un processus. Le discours nous persuade que l'ensemble des actions entreprises en 1995 n'a de signification qu'en regard d'un processus dont l'achèvement 
est reporté dans un avenir temporellement indéterminé. Toutefois, cet avenir est investi d'un sens plutôt favorable : «C'est vraiment une année charnière pour Bell. Et nous sommes prêts.»

La crédibilité de cet exercice de prospective ne serait pas la même si le destinataire présumé des propos ne partageait pas la vision néolibérale de la gestion d'entreprise qui imprègne ici le discours. L'efficacité d'une argumentation est en rapport avec son degré de prévisibilité. Dans un monde corporatif qui croit dans la validité et dans l'efficacité d'une gestion dont les mots-clés sont restructuration, investissements technologiques et prise en compte de la mondialisation de l'économie, il est certain que le recours à ces thématiques favorise l'obtention d'un jugement d'approbation.

Lédifice de l'argumentation est donc constitué surtout des matériaux suivants : définition d'un objet thématique principal porteur de jugements favorables plausibles, structure narrative mettant l'accent sur la qualification du sujet et faisant ressortir tout son potentiel dynamique et guerrier, recours à l'énumération de type finie, achevée, pour renforcer l'impression de contrôle de l'entreprise sur le processus mis en train et, enfin, exploitation thématique de valeurs considérées comme vraies par le destinataire du discours.

\section{Seagram, Rapport annuel, 1994}

À l'instar d'Hydro-Québec et de Bell Canada, Seagram constate que ses ventes augmentent mais que les profits diminuent. Qu'à cela ne tienne, l'incipit du texte de la direction s'apparente à celui d'Hydro-Québec et consiste en un jugement conclusif très clairement favorable à l'entreprise, quoique rédigé dans une langue médiocre, traduite de l'anglais : «En 1994, Seagram a dominé la concurrence, en confortant ses marques et ses marchés dans le monde entier. La Compagnie a alors pris la décision de se surpasser et adopté les moyens pour y arriver.»

Voilà un syllogisme incomplet classique (enthymème), dont la prémisse non exprimée est l'opinion commune selon laquelle toute entreprise cherche constamment à relever de nouveaux défis d'affaires. Cette prémisse implicite est suggérée par les mots utilisés et favorise la lecture du message selon l'intention de l'énonciateur. La proposition est un jugement d'évaluation de la performance d'entreprise sur le plan des ventes, excluant la prise en compte des bénéfices. La conclusion est déduite de la proposition et le choix des mots favorise le détournement de sens. Ce qui, en réalité, correspond à la conclusion de l'adoption d'un plan de redressement est présenté comme la décision de se surpasser, ce qui suppose une situation initiale satisfaisante et un sujet ayant la 
pleine maîtrise de la situation. En clair, il faudrait lire plutôt: Seagram a augmenté ses ventes, mais le bénéfice a chuté. Conséquemment, l'entreprise a élaboré un plan de redressement.

Cette rhétorique permet, dans le premier paragraphe, de donner à l'entreprise un rôle de sujet libre et entièrement maître de la situation. La stratégie de Seagram consiste à faire valoir les résultats par anticipation de son plan de redressement (appelé ensemble de moyens pour se surpasser) en les donnant comme conclusion inévitablement favorable d'actions déjà accomplies ou en train de s'accomplir. Sous l'action de la rhétorique, l'évaluation est transformée en observation : les jugements de valeur deviennent jugements de faits.

Cet objet thématique tronqué par le discours est ensuite développé par addition de faits : l'énumération des actions sert de validation à un jugement global porté sur des résultats donnés comme le prolongement logique du programme réalisé. Aux faits cumulés en termes de ventes de produits (donc vérifiables), le texte associe des décisions de gestion, dont la justification et l'effet sont évidemment moins facilement comptabilisables. Tous ces exemples de bonne performance s'additionnent pour créer un effet dynamique et sont ponctués de jugements d'évaluation favorables, prenant appui sur des données chiffrées, absolues et relatives. La rhétorique du nombre et du fait réel joue ici sa pleine fonction persuasive.

En additionnant les réalisations sur le plan des ventes aux décisions de gestion, plus intangibles, et imbriquées dans des processus non achevés, on obtient une impression malgré tout favorable de l'année écoulée, en vertu du raisonnement par analogie. Il n'y a pas ici de doute quant au retour anticipé des bénéfices : l'énonciateur pose le sujet du programme comme en pleine possession de ses moyens.

Contrairement à ce qui était le cas dans le rapport de Bell Canada, mais à l'instar d'Hydro-Québec, Seagram donne à voir sa faiblesse comme le signe de sa force en émergence. Une structure argumentative serrée, la dissémination de verbes de jugements conclusifs, des parties narratives axées sur la sanction favorable anticipée et qui ne mettentjamais en doute la qualification du sujet de l'action et, enfin, une habile utilisation de l'énumération assortie d'arguments chiffrés caractérisent la stratégie de validation dans ce texte.

\section{En guise de conclusion}

Il apparaît donc que, pour établir la vérité de ses propos, l'énonciateur du discours comprenant une mauvaise nouvelle a recours à divers procédés rhétoriques. 
La thématisation de l'objet développé dans le discours est capitale : comment est-il nommé, comment est-il prédiqué? Ce choix thématique, on l'a vu, permet des détournements de sens aux multiples possibilités selon les intentions de l'énonciateur. Le procédé est largement utilisé en rédaction administrative et ouvre la perspective de voir émerger dans l'analyse un répertoire de lieux communs, ou topoï, car l'énonciateur cherche avec détermination le moyen de réinstaller dans son discours un réseau de lexèmes ou syntagmes figés, porteurs de significations réjouissantes, et qui servent de points de repère au destinataire du message. La thématisation de l'objet devient ainsi un exercice de raccrochage à des objets langagiers valorisés, peu définis car assimilés à des évidences, ce qui renforce leur caractère persuasif.

Le choix d'une macrostructure textuelle s'avère d'importance considérable pour la production d'un effet de vérité. Si la structure est de type narratif, l'accent est mis sur la requalification du sujet, donc sur le développement de la phase d'acquisition de compétence du sujet. L'énumération des qualités acquises ou en voie de l'être tient alors lieu de preuve de leur validité. Lorsque l'enveloppe textuelle devient argumentative, le raisonnement syllogistique domine : une proposition sous forme de jugement d'évaluation global, formulée de manière à paraître un jugement d'observation, permet de déduire avec aisance une conclusion favorable à l'émetteur du jugement. Cette constatation confirme l'analyse de M. Angenot (1989 : 159), selon qui : «Toute entreprise rhétorique structure le réel en le schématisant, elle se voue à interpréter le monde en vue de le transformer, à donner une unité et un sens (signification et orientation) aux expériences vécues, par nature hétérogènes, de l'auditoire qu'elle se construit."

Lorsque les résultats positifs d'une quelconque entreprise ne sont qu'escomptés, le risque à éviter est que toute amélioration pressentie soit exprimée, dans le discours, au temps futur, le présent et le passé composé étant réservés à l'expression de l'échec ou de l'incertitude. Pour contourner cet obstacle, l'énonciateur ne développe que le récit des succès de l'entreprise et met en lumière la liste des actions de requalification du sujet, actions achevées, et dont l'énumération joue ici pleinement son rôle d'outil persuasif. Alors son récit se déroule au présent et au passé. L'énonciateur peut également rétrécir l'angle de vision du destinataire en substituant au récit de l'année écoulée la justification d'un jugement favorable sur le bilan de l'entreprise, justification articulée essentiellement sur l'addition de faits et de jugements interreliés. Encore là, le discours se déroule au présent et au passé.

Enfin, dernière remarque sur les stratégies persuasives à l'oeuvre dans le discours administratif, il est notable que, dans ce genre d'exercice persuasif, la 
sobriété souvent attribuée au milieu administratif cède la place à un discours fortement appréciatif et évaluatif, ce qui apparaît une stratégie efficace dans le contexte. Le message de la direction est fortement orienté vers son effet appréhendé sur le destinataire.

\section{Bibliographie}

Angenot, M. (1989). La propagande socialiste, éléments de rhétorique et de pragmatique, Texte no 8/9, Presses de l'Université de Toronto.

Barthes, R. (1970). L'ancienne rhétorique, Communications no 16, Seuil.

Charaudeau, P. (1995). Une analyse sémiolinguistique du discours, Langages no 117, Les analyses du discours en France, Larousse.

Kerbrat-Orecchioni, C. (1981). Argumentation et mauvaise foi, L'argumentation, Presses universitaires de Lyon.

Moirand, S. (1995). L'évaluation dans les discours scientifiques et professionnels, Les enjeux des discours spécialisés, Presses de la Sorbonne nouvelle.

Oléron, P. (1993). L'Argumentation, Presses universitaires de France.

Perelman, Ch. (1970). Le champ de l'argumentation, Presses universitaires de Bruxelles.

Perelman, Ch. (1988). L'empire thétorique, J.Vrin.

Vignaux, Georges; Lascoumes, Pierre; Moreau-Capdevielle, G. (1978). $l_{\gamma}$ a parmi nous des monstres, Communications no 28, Seuil.

Vignaux, Georges (1988). Le discours acteur du monde, Ophrys.

\section{Rapports annuels cités}

Bell Canada (1995).

Hydro-Québec (1994).

Seagram (1994). 